\title{
CT Perfusion Can Predict Overexpression of CXCL8 (Interleukin-8) in Head and Neck Squamous Cell Carcinoma
}

\author{
S.Y. Jo, P.I. Wang, J.E. Nör, E.L. Bellile, Z. Zhang, F.P. Worden, A. Srinivasan, and S.K. Mukherji
}

\begin{abstract}
BACKGROUND AND PURPOSE: Increased angiogenesis in head and neck squamous cell carcinoma correlates to more aggressive tumors with increased morbidity. Because both elevated blood flow and high serum CXCL8 levels are correlated with increased angiogenesis, our objective was to see if elevated blood flow measured with CT perfusion correlated with CXCL8 levels, thereby helping to identify candidates for targeted therapies that inhibit the Bcl-2 proangiogenic pathway associated with CXCL8.
\end{abstract}

MATERIALS AND METHODS: Seven patients with locally recurrent or metastatic head and neck squamous cell carcinoma were enrolled in the trial. These patients underwent CT perfusion and the following parameters were measured: blood volume, blood flow, capillary permeability, and MTT; relative values were calculated by dividing by normal-appearing muscle. Serum was drawn for CXCL8 enzymelinked immunosorbent assay analysis in these patients.

RESULTS: There was a significant positive correlation between the CXCL8 levels and relative blood flow $(r=0.94 ; P=.01)$. No correlation was found between CXCL8 and relative blood volume, relative capillary permeability, or relative MTT.

CONCLUSIONS: Relative blood flow may be useful as a surrogate marker for elevated CXCL8 in patients with head and neck squamous cell cancer. Patients with elevated relative blood flow may benefit from treatment targeting the Bcl-2 proangiogenic pathways.

ABBREVIATIONS: $\mathrm{BF}=$ blood flow; $\mathrm{BV}=$ blood volume; $\mathrm{CP}=$ capillary permeability; $\mathrm{HNSCC}=$ head and neck squamous cell carcinoma; $\mathrm{VEGF}=$ vascular endothelial growth factor

S tudies in patients with cancer have found that increased blood flow on CT perfusion is correlated with angiogenesis and increased tumor vascularity, and increased tumor vascularity is associated with local recurrence and metastasis. ${ }^{1-7}$ Therefore, increased blood flow on CTP suggests that a tumor is more aggressive and more likely to result in poor outcome.

A serum chemokine, CXCL8 or interleukin-8, also predicts poor patient survival because this pro-angiogenic chemokine

Received January 23, 2013; accepted after revision March 16.

From the Departments of Radiology (S.Y.J., A.S., P.I.W., S.K.M.), Otolaryngology (J.E.N.), and Medical Oncology (F.P.W.), and Cancer Center Biostatistics Unit (E.L.B.), University of Michigan Hospital, Ann Arbor, Michigan; and Angiogenesis Research Laboratory (J.E.N., Z.Z.), Department of Restorative Sciences, University of Michigan School of Dentistry, Ann Arbor, Michigan.

Support for this project was provided by grant P50-CA-97248 (University of Michigan Head and Neck SPORE) from the $\mathrm{NIH} / \mathrm{NCl}$ and grant R01-DE23220 from the NIH/NIDCR.

Please address correspondence to Suresh K. Mukherji, MD, Department of Radiology, B2 Room A209, University of Michigan, 1500 East Medical Center Dr, Ann Arbor, MI 48109; e-mail: mukherji@umich.edu

- Indicates open access to non-subscribers at www.ajnr.org

Indicates article with supplemental on-line figure

http://dx.doi.org/10.3174/ajnr.A3610 promotes metastasis by neutrophil infiltration and stimulates vascular endothelial cell proliferation, survival, and migration. ${ }^{8-10}$ CXCL8 is an end-product of the vascular endothelial growth factor (VEGF) pathway, a key pathway for angiogenesis (Fig 1). Inhibition of the VEGF pathway is a widely pursued strategy to curtain angiogenesis, and at our institution, the experimental drug AT-101 is being used to inhibit Bcl-2, which is an upstream molecule in the VEGF pathway. ${ }^{11-13}$

Our goal was to see if CTP-derived parameters correlate with the level of CXCL8 in patient serum. Because CXCL8 is not currently clinically orderable, CTP could potentially serve as a surrogate marker for patient prognosis and/or anti-angiogenesis treatment patient selection.

\section{MATERIALS AND METHODS Human Subjects}

Seven patients were prospectively enrolled in an institutional review board-approved phase II clinical trial of docetaxel (Taxotere) and the investigational drug AT-101 for locally recurrent or metastatic head and neck squamous cell carcinoma (HNSCC). The patients underwent both pretreatment CTP and serum measurements of CXCL8 before the initiation of AT-101. Inclusion 
criteria were men and nonpregnant, nonlactating women $>18$ years old, confirmed diagnosis of stage IVC (metastatic) or advanced, locally recurrent HNSCC not amenable to surgery or palliative radiation therapy.

\section{Serum Samples and Enzyme-Linked Immunosorbent Assay}

After patient consent, peripheral blood was collected. Blood samples were incubated for 2 hours at room temperature for clot formation before centrifuging for 20 minutes at approximately $1000 \mathrm{~g}$. Then, serum was removed and either assayed immediately or aliquoted and stored at $-80^{\circ} \mathrm{C}$.

Expression levels of CXCL8 were determined by enzymelinked immunosorbent assay (Quantikine; R\&D Systems, Minneapolis, Minnesota) according to the manufacturer's instructions. After the development of the colorimetric reaction, the optical attenuation was measured on a microplate spectrophotometer, Tecan GENios (MTX Lab Systems, Vienna, Virginia) at a wavelength of $450 \mathrm{~nm}$; the concentration $(\mathrm{pg} / \mathrm{mL})$ was derived from optical density readings and a generated standard curve. Each sample was tested in triplicate wells.

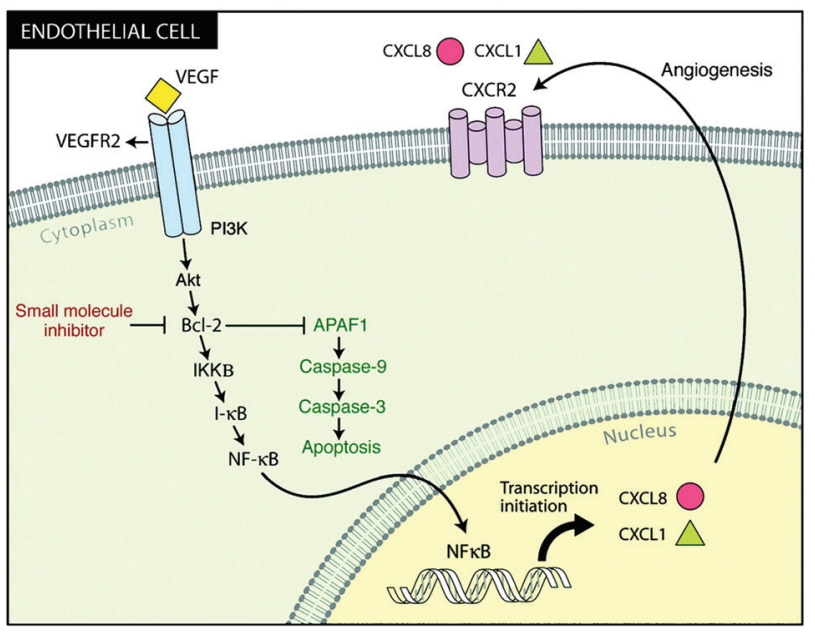

FIG 1. VEGF pathway for angiogenesis. $\mathrm{Bcl}-2$, in the beginning of the pathway, is inhibited by a small molecule inhibitor, AT-101. The downstream serum marker CXCL8 is depicted by the red circle. Reprinted with permission from the American Association for Cancer Research: Karl E, Warner K, Zeitlin B, et al. Bcl-2 Acts in a Proangiogenic Signaling Pathway through Nuclear Factor-kB and CXC Chemokines. Cancer Res 2005;65:5063-69.

\section{CT Perfusion}

All 7 patients underwent CTP (LightSpeed Ultra; GE Healthcare, Milwaukee, Wisconsin). The technique consisted of injecting 50 $\mathrm{mL}$ of nonionic contrast (4 mL/s; Ultravist 300; Bayer HealthCare Pharmaceuticals, Wayne, New Jersey). At 5 seconds into the injection, a cine acquisition was initiated with the following parameters: $120 \mathrm{kV}$ and $60 \mathrm{~mA}$. The cine images covered a $4-\mathrm{cm}$ area, as 8 images were taken with a $5-\mathrm{mm}$ section thickness per cine rotation. A region of interest was placed in the internal carotid artery to generate the contrast enhancement curve. A single observer (a fellowship-trained neuroradiologist with 20 years' experience in head and neck imaging), who was aware of the tumor site but unaware of CXCL8 levels, drew regions of interest $\left(25-30 \mathrm{~mm}^{2}\right)$ through the tumor and in normal-appearing muscle (Figs 2 and 3). Streak and other artifacts were avoided. The perfusion data were postprocessed by a deconvolution-based method into maps that represented blood volume (BV), blood flow (BF), capillary permeability (CP), and MTT, by use of the commercially available Perfusion-4 software package on an Advantage Windows Workstation (GE Healthcare).

The relative ( $r$ ) CTP measures were calculated by dividing our $\mathrm{BF}, \mathrm{BV}, \mathrm{CP}$, and MTT values by the normal-appearing tissue values to create the rBF, rBV, rMTT, and rCP.

\section{Statistics}

Pearson and Spearman correlation coefficients were calculated to assess the relationship between the pretreatment serum CXCL8 values and CTP measures. Raw (BF, BV, MTT, CP) and relative (rBF, rBV, rMTT, rCP) CTP measures were analyzed.

Statistical significance of the correlations was computed by use of 2 -sided exact tests. Values of $P<.05$ were considered statistically significant. No corrections for multiple testing were used because of the exploratory nature of this preliminary analysis and because the CTP measures are unlikely to be independent. All statistical analysis was performed in SAS software version 9.3 (SAS Institute, Cary, North Carolina).

\section{RESULTS}

Of the 7 patients, $86 \%$ were men (6/7), $71 \%$ had metastatic disease (5/7), 29\% had locally recurrent disease (2/7), and 86\% (6/7) had died of their disease since enrollment (3-16 months from enrollment). The average age was 59 years (range, 41-73 years), and
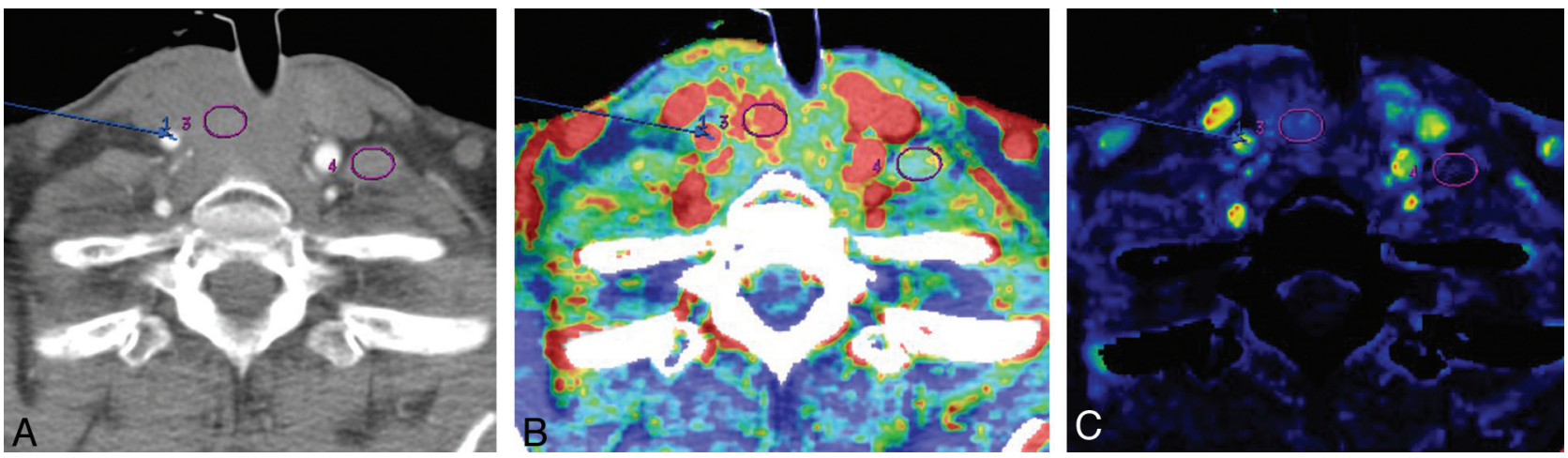

FIG 2. Peri-stomal recurrence of HNSCC (patient 5) in a 73-year-old woman. A, Base image shows arterial enhancement. Blue arrow points to the right internal carotid artery (for the arterial input signal). Circle 3 is within the tumor; circle 4 is in contralateral noninvolved musculature. B, Blood volume image shows that the region of interest (circle 3) was placed in solid non-necrotic tumor. C, Blood flow imaging shows minimally increased signal within the tumor bed (circle 3). 

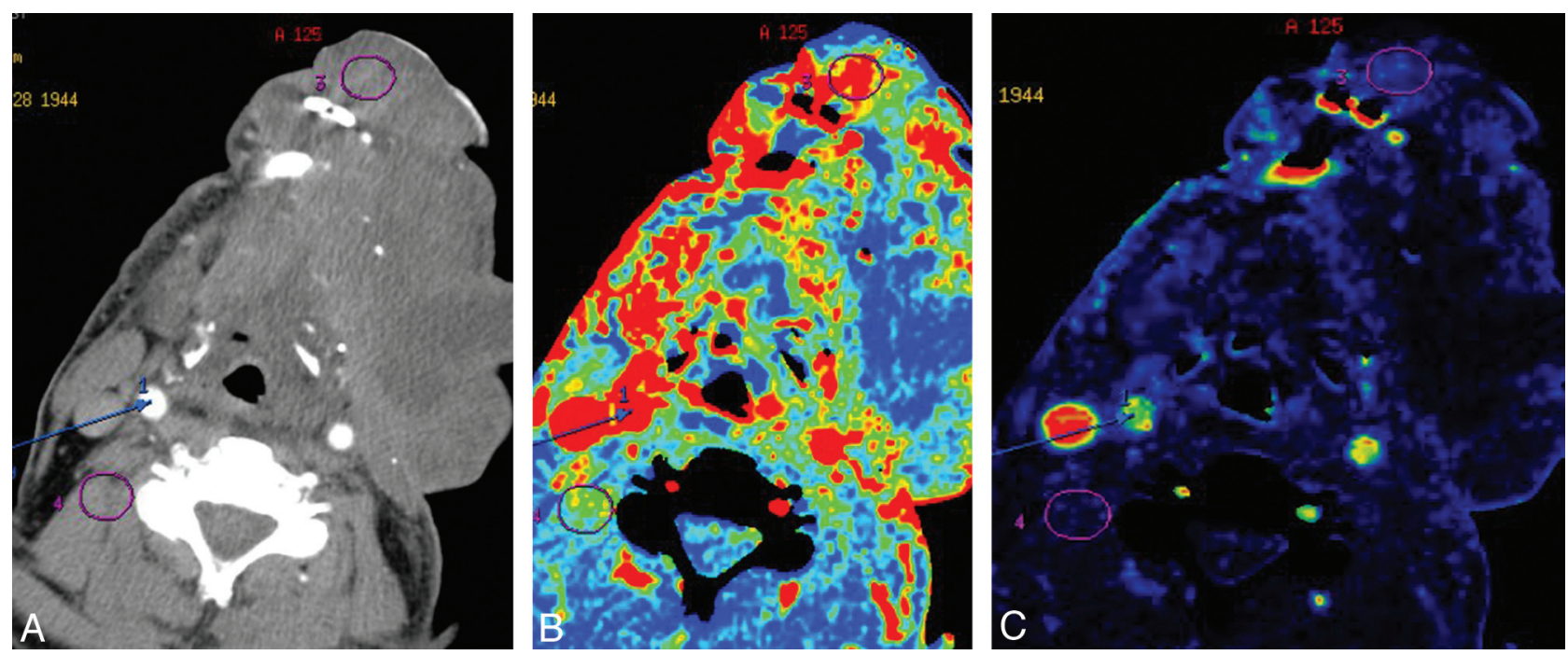

FIG 3. Floor-of-the-mouth HNSCC in a 67-year-old man (patient 2). A, Base image shows arterial enhancement. Blue arrow points to the right internal carotid artery (for the arterial input signal). Circle 3 is within the tumor; circle 4 is in noninvolved musculature. B, Blood volume image shows that the region of interest (circle 3 ) within the tumor was placed in solid non-necrotic tumor. C, Blood flow imaging shows minimally increased signal within the tumor bed (circle 3).

Table 1: Patient data, tumor characteristics, and clinical course in 7 patients with advanced, treatment-resistant HNSCC

\begin{tabular}{|c|c|c|c|c|c|c|c|}
\hline Patient & Location & Race & Sex & Age & Metastatic Site & Tobacco & $\begin{array}{l}\text { Clinical } \\
\text { Course }\end{array}$ \\
\hline 1 & Right nasal cavity & White & Male & 54 & $\mathrm{n} / \mathrm{a}$ & None & Died \\
\hline 2 & Left floor of mouth & White & Male & 67 & $\mathrm{n} / \mathrm{a}$ & 50 Pack-years & Died \\
\hline 3 & Right parotid gland & African American & Male & 68 & Lung & 30 Pack-years & Alive \\
\hline 4 & Left oral tongue & White & Male & 57 & Lung, liver, lymph nodes, bone & None & Died \\
\hline 5 & Peri-stomal & African American & Female & 73 & Lung & 20 Pack-years & Died \\
\hline 6 & Peri-stomal & White & Male & 54 & Lung & 60 Pack-years & Alive \\
\hline 7 & Left floor of mouth & White & Male & 41 & Lung, lymph nodes & 15 Pack-years & Alive \\
\hline
\end{tabular}

$71 \%(5 / 7)$ had significant smoking history. Patient demographics are summarized in Table 1.

Pearson correlation showed a statistically significant positive correlation between CXCL8 expression level and $\mathrm{rBF}(r=0.94$, $P=.01$; Fig 4 ). Spearman rank correlation coefficient also showed positive correlation between CXCL8 and $\mathrm{rBF}$, though statistical significance was not reached $(r=0.61, P=.17)$. No significant correlation was found between CXCL8 and BV, rBV, CP, rCP, MTT, or rMTT as summarized in Table 2. The scatter plot for these variables can be seen in the On-line Figure. The mean, standard deviation, and range of the CTP parameters and CXCL8 expression levels in the 7 patients studied are summarized in Table 3.

\section{DISCUSSION}

Angiogenesis is the mechanism by which new capillaries form from existing vasculature and is fundamental to normal tissue/ organ growth and development. ${ }^{14}$ Hypermitotic cellular activity requires the formation of new blood vessels, leading to tumor neovascularity. ${ }^{14}$ Previous investigators have shown that tumors cannot expand $>0.1-0.2 \mathrm{~mm}$ in size without the induction of angiogenesis. ${ }^{14}$ Three of the primary activators of angiogenesis that promote endothelial proliferation are VEGF, CXCL1 (growth-related oncogene, GRO- $\alpha$ ), and CXCL8 (interleukin8). ${ }^{15,16}$ The expression of CXCL8 is activated by the nuclear factor- $\kappa \mathrm{B}$ signaling pathway, which is controlled by a pro-angiogenic signaling molecule, $\mathrm{Bcl}-2 .{ }^{17}$ Recent studies have shown that $\mathrm{Bcl}-2$

\section{Blood Flow and CXCL8}

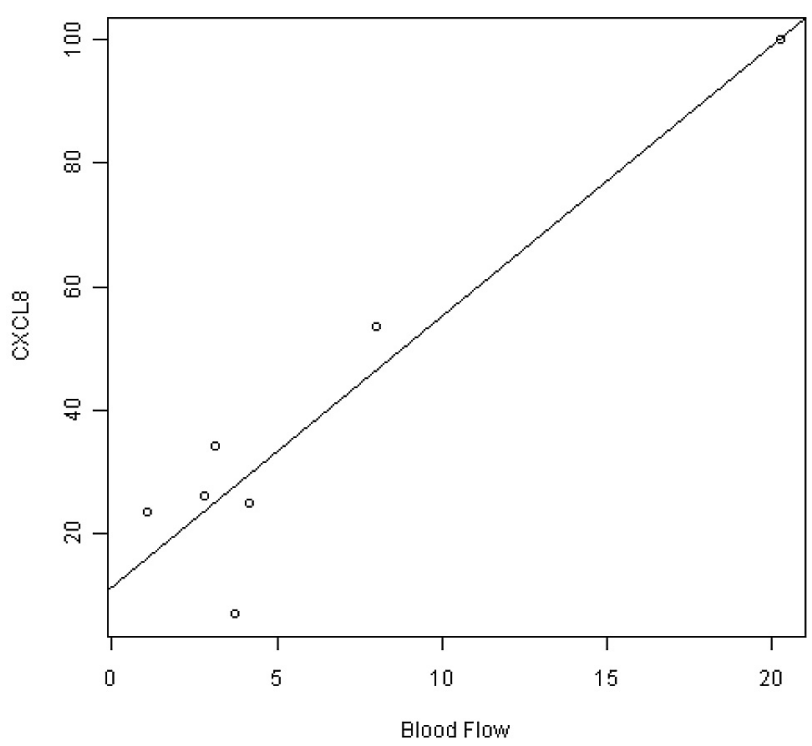

FIG 4. Scatterplot displays positive correlation between serum CXCL8 expression level $(\mathrm{pg} / \mathrm{mL})$ and $\mathrm{rBF}(\mathrm{mL} / 100 \mathrm{~g}$ per minute) in the 7 patients with advanced stage HNSCC.

is substantially upregulated in HNSCC compared with normal oral mucosal endothelial cells, which would suggest that HNSCC may have elevated levels of CXCL8. ${ }^{18}$ 
Table 2: Pearson correlation between serum CXCL8 expression level and CTP parameters

\begin{tabular}{ccc} 
Parameter & $\begin{array}{c}\text { CXCL8 Correlation } \\
\text { Coefficient }\end{array}$ & P Value \\
\hline BF & -0.16 & .67 \\
BV & 0.22 & .47 \\
CP & -0.57 & .19 \\
MTT & -0.03 & .92 \\
rBF & 0.94 & .01 \\
rBV & -0.14 & .77 \\
rCP & -0.07 & .88 \\
rMTT & 0.18 & .68 \\
\hline
\end{tabular}

Table 3: CTP and CXCL8 expression results in 7 patients

\begin{tabular}{lcc}
\hline Parameter & Mean \pm SD & Range \\
\hline CXCL8 & $38.46 \pm 30.46$ & $6.97-99.89$ \\
BF & $74.08 \pm 56.41$ & $14.78-187.93$ \\
BV & $22.56 \pm 35.96$ & $5.08-103.71$ \\
CP & $48.07 \pm 21.56$ & $16.73-79.84$ \\
MTT & $9.46 \pm 2.08$ & $7.34-13.81$ \\
rBF & $6.18 \pm 6.56$ & $1.08-20.26$ \\
rBV & $2.77 \pm 0.87$ & $1.31-3.72$ \\
rCP & $3.31 \pm 1.88$ & $1.46-6.70$ \\
rMTT & $1.12 \pm 0.41$ & $0.78-1.94$ \\
\hline
\end{tabular}

CXCL8 is known to be a promoter of angiogenesis and a regulator of cell growth and motility in HNSCC. ${ }^{18}$ Overexpression of CXCL8 in HN4 cells (a primary HNSCC cell line with low endogenous CXCL8) increased cell growth and enhanced migration, whereas RNAi-mediated knockdown of CXCL8 expression in HN12 cells (a metastatic HNSCC cell line with elevated CXCL8) decreased proliferation and inhibited invasion through a basement membrane substrate. ${ }^{19}$

Our results show a significant linear positive correlation between patient serum CXCL8 levels and $\mathrm{rBF}$ values $(r=0.94$; $P=.01$ ), which suggests that an association exists between serum biomarker CXCL8 and rBF values. This study further supports the growing concept that noninvasive imaging biomarkers can be predictive of molecular biomarkers. In HNSCC, CTP has been shown to predict microvascular attenuation and epidermal growth factor receptor overexpression and to correlate with patient outcomes in patients undergoing induction chemotherapy. $1,20-22$

This preliminary study suggests that CTP can predict the expression of small, biologically active proteins that are important end products on the VEGF proangiogenic pathway. The capability of CTP to predict levels of these angiogenic proteins suggests that CTP may help to identify highly vascular and more aggressive tumors through its ability to predict increased microvascular attenuation and the overexpression of CXCL8. Such tumors could be potential candidates for anti-angiogenic agents such as AT-101.

There are several limitations to our study. Our data were prospectively obtained in a small number of patients from a single institution. Statistical significance was tested by use of exact tests to address the issue of small sample size, but the potential for outliers to have heavy influence exists. We performed the same analysis excluding a potential outlier patient with the highest CXCL8 and $\mathrm{rBF}$, and, as expected, we observed a weaker correlation than reported for all 7 patients. We could find no clinical reason for ex- cluding this patient from our reported results but acknowledge that further study with a larger, more robust sample is necessary.

If future studies show that the correlation between CXCL8 and rBF holds in patients with all stages of HNSCC (not only stage IV as we describe), then CTP could become a useful, noninvasive tool for predicting treatment response. Because patients with HNSCC are routinely imaged to evaluate treatment response, CTP would provide complementary information to help guide therapy.

\section{CONCLUSIONS}

Our preliminary results show a significant linear positive correlation with patient serum CXCL8 level and rBF values in advanced HNSCC, which suggests that an association exists between serum biomarker CXCL8 and rBF values.

Disclosures: Jacques Nör-RELATED: Grant: $\mathrm{NIH}^{*}{ }^{*}$ Comments: University of Michigan Head and Neck SPORE; UNRELATED: Grants/Grants Pending: Medimmune, * Ascenta.* Emily Bellile—RELATED: Grant: Head and Neck SPORE grant, ${ }^{*}$ Comments: Part of my salary is paid for through funds from the University of Michigan Head and Neck SPORE Grant. Francis Worden—UNRELATED: Expert Testimony: I was involved in reviewing a legal case for a woman in NC with an advanced laryngeal cancer. I provided a deposition and gave an expert testimony in court; Payment for Lectures (including service on Speakers Bureaus): Bristol-Myers-Squibb ( ${ }^{*}$ money paid to institution).

\section{REFERENCES}

1. Ash L, Teknos TN, Gandhi D, et al. Head and neck squamous cell carcinoma: CT perfusion can help noninvasively predict intratumoral microvessel density. Radiology 2009;251:422-28

2. Delrue L, Blanckaert P, Mertens D, et al. Assessment of tumor vascularization in pancreatic adenocarcinoma using 128-slice perfusion computed tomography imaging. J Comput Assist Tomogr 2011;35:434-38

3. Afaq A, Akin O. Imaging assessment of tumor response: past, present and future. Future Oncol 2011;7:669-77

4. Jiang HJ, Zhang ZR, Shen BZ, et al. Quantification of angiogenesis by CT perfusion imaging in liver tumor of rabbit. Hepatobiliary Pancreat Dis Int 2009;8:168-73

5. Gleich LL, Biddinger PW, Pavelic ZP, et al. Tumor angiogenesis in T1 oral cavity squamous cell carcinoma: role in predicting tumor aggressiveness. Head Neck 1996;18:343-46

6. Kupisz K, Chibowski D, Klatka J, et al. Tumor angiogenesis in patients with laryngeal cancer. Eur Arch Otorhinolaryngol 1999;256: 303-05

7. Lentsch EJ, Goudy S, Sosnowski J, et al. Microvessel density in head and neck squamous cell carcinoma primary tumors and its correlation with clinical staging parameters. Laryngoscope 2006; 116:397-400

8. Waugh DJ, Wilson C. The interleukin-8 pathway in cancer. Clin Cancer Res 2008;14:6735-41

9. Trellakis S, Bruderek K, Dumitru CA, et al. Polymorphonuclear granulocytes in human head and neck cancer: enhanced inflammatory activity, modulation by cancer cells and expansion in advanced disease. Int J Cancer 2011;129:2183-93

10. De Larco JE, Wuertz BR, Furcht LT. The potential role of neutrophils in promoting the metastatic phenotype of tumors releasing interleukin-8. Clin Cancer Res 2004;10:4895-900

11. Pourgholami MH, Morris DL. Inhibitors of vascular endothelial growth factor in cancer. Cardiovasc Hematol Agents Med Chem 2008;6:343-47

12. Liu Q, Wang HG. Anti-cancer drug discovery and development: Bcl-2 family small molecule inhibitors. Commun Integr Biol 2012;5: $557-65$ 
13. Zeitlin BD, Zeitlin IJ, Nor JE. Expanding circle of inhibition: smallmolecule inhibitors of Bcl-2 as anticancer cell and antiangiogenic agents. J Clin Oncol 2008;26:4180-88

14. Carmeliet P, Jain RK. Angiogenesis in cancer and other diseases. Nature 2000;407:249-57

15. Mehrad B, Keane MP, Strieter RM. Chemokines as mediators of angiogenesis. Thromb Haemost 2007;97:755-62

16. Singh S, Sadanandam A, Singh RK. Chemokines in tumor angiogenesis and metastasis. Cancer Metastasis Rev 2007;26:453-67

17. Nor JE, Christensen J, Mooney DJ, et al. Vascular endothelial growth factor (VEGF)-mediated angiogenesis is associated with enhanced endothelial cell survival and induction of Bcl-2 expression. Am J Pathol 1999;154:375-84

18. Kaneko T, Zhang Z, Mantellini MG, et al. Bcl-2 orchestrates a crosstalk between endothelial and tumor cells that promotes tumor growth. Cancer Res 2007;67:9685-93
19. Christofakis EP, Miyazaki H, Rubink DS, et al. Roles of CXCL8 in squamous cell carcinoma proliferation and migration. Oral Oncol 2008;44:920-26

20. Hoefling NL, McHugh JB, Light E, et al. Human papillomavirus, p16, and epidermal growth factor receptor biomarkers and CT perfusion values in head and neck squamous cell carcinoma. AJNR Am J Neuroradiol 2013;34:1062-66

21. Zima A, Carlos R, Gandhi D, et al. Can pretreatment CT perfusion predict response of advanced squamous cell carcinoma of the upper aerodigestive tract treated with induction chemotherapy? AJNR Am J Neuroradiol 2007;28:328-34

22. Surlan-Popovic K, Bisdas S, Rumboldt Z, et al. Changes in perfusion CT of advanced squamous cell carcinoma of the head and neck treated during the course of concomitant chemoradiotherapy. AJNR Am J Neuroradiol 2010;31:570-75 